\title{
The possible role of vortex shedding in the excitation of kink-mode oscillations in the solar corona
}

\author{
V. M. Nakariakov ${ }^{1}$, M. J. Aschwanden ${ }^{2}$, and T. Van Doorsselaere ${ }^{1}$ \\ 1 Physics Department, University of Warwick, Coventry, CV4 7AL, UK \\ e-mail: V.Nakariakov@warwick.ac.uk \\ 2 Solar \& Astrophysics Laboratory, Lockheed Martin Advanced Techology Center, Palo Alto, CA 94304, USA \\ Received 22 August 2008 / Accepted 22 April 2009
}

ABSTRACT

\begin{abstract}
We propose a model for the excitation of horizontally polarised transverse (kink) magnetoacoustic oscillations of solar coronal loops by upflows associated with coronal mass ejections. If the magnetic field in the plasma that is dragged in the vertical direction by the flow is parallel to the loop, the phenomenon of vortex shedding causes the appearance of a quasi-periodic horizontal force that is applied to alternating sides of the loop. The period of the force is determined by the flow speed and the loop's minor radius. The oscillations are excited the most effectively when the force is in resonance with the natural frequency of the kink oscillations. This model can explain the selectivity of the excitation of the oscillations and the initial growth of the oscillation amplitude.
\end{abstract}

Key words. waves - Sun: corona - Sun: coronal mass ejections (CMEs) - magnetohydrodynamics (MHD)

\section{Introduction}

Transverse (or kink) oscillations of solar coronal loops is perhaps one of the most intensively studied examples of solar coronal wave activity (see Aschwanden 2004; Nakariakov \& Verwichte 2005). The oscillations are usually seen with the TRACE images as impulsively excited transverse periodic displacements of EUV loops (Aschwanden et al. 1999; Nakariakov et al. 1999). The typical periods of transverse oscillations are about several minutes, varying among different loops. A characteristic feature of these oscillations is strong damping, within a few oscillation periods. In the majority of observed cases, the polarisation plane of the oscillations is horizontal, or parallel to the solar surface. Usually, only the global (or fundamental) mode of oscillation is observed, with the maximum displacement near the loop apex and nodes at the loop footpoints. In all cases, transverse oscillations were observed in association with filament destabilisation or coronal mass ejections (Schrijver et al. 2002). A detailed summary of observational properties of transverse oscillations obtained with TRACE is given in Aschwanden et al. (2002) and Schrijver et al. (2002). Recently, the Doppler shift variations of EUV emission lines were also attributed to kink mode oscillations (Van Doorsselaere et al. 2008b).

Transverse oscillations of coronal loops are interpreted as oblique fast magnetoacoustic waves guided by a fieldaligned Alfvén speed inhomogeneity (see, e.g., discussion in Van Doorsselaere et al. 2008a). The popular theoretical model for the description of transverse oscillations is the straight cylinder model developed by Edwin \& Roberts (1983), which links observed properties of the oscillations with the parameters of the plasma in and out of the oscillating loop. In particular, the period of transverse oscillations is determined by the magnetic field, and the densities of the plasma inside and outside the loop. This makes transverse oscillations an excellent tool for estimating the magnetic field (Nakariakov \& Ofman 2001).
The mechanism for the excitation of transverse loop oscillations is still not understood. One widely accepted option is excitation of the oscillations by a flare-generated blast wave (Nakariakov et al. 1999; Ofman 2007). In this scenario, the flaring energy release creates a fast magnetoacoustic pulse (a blast wave) propagating from the flare site almost isotropically, which in turn hits a loop and excites the oscillations in the plane, parallel to the fast wave vector. Remaining a plausible option, this mechanism does not explain several important observational features of this phenomenon. In particular, it is not clear (1) the cause of the selectivity of the excitation; (2) the reason some loops respond to excitation, while the majority of them do not; (3) the preferred excitation of the global mode, while one would expect an impulsive, broadband driver to excite a number of modes; (4) the dominance of horizontal polarisation; and (5) the reason, in a number of cases, the oscillation is initially growing during a few periods (e.g. 1a, 4a, 5c, 7a, 10a, 15a, 16a, and, marginally, 3a and 8a of Aschwanden et al. 2002).

Often, transverse oscillations of coronal loops are observed in association with coronal mass ejections (CME). For example, Hudson \& Warmuth (2004) points out that 12 out of 28 oscillatory events were accompanied with type II bursts, a characteristic signature of CME. A similar conclusion has been reached by Wills-Davey (2007), who analysed TRACE data. So far, the possible role of the CME upflows in the excitation of transverse loop oscillations has not received proper attention. We discuss the mechanism for the excitation of horizontally-polarised transverse oscillations, based upon the interaction of loops with CME-induced vertical flows due to the effect of vortex shedding.

\section{Observational evidence of the link between oscillations and CMEs}

There is observational evidence that transverse oscillations are associated with the eruptions of coronal plasmas. Already in a 
Table 1. Association of 17 loop oscillation events with flare and CME events.

\begin{tabular}{|c|c|c|c|c|c|c|}
\hline $\begin{aligned} \text { Event } \\
\text { Nr. }\end{aligned}$ & Date & $\begin{array}{l}\text { TRACE movie } \\
\text { start/end time }\end{array}$ & $\begin{array}{l}\text { NOAA } \\
\text { AR }\end{array}$ & $\begin{array}{l}\text { GOES } \\
\text { flare }\end{array}$ & $\begin{array}{l}\text { GOES flare } \\
\text { start/end time }\end{array}$ & $\begin{array}{l}\text { SOHO/LASCO } \\
\text { CME detection time, type, linear speed }\end{array}$ \\
\hline 1 & 14 Jul. 1998 & $12: 45-13: 44$ & 8270 & M4.6 & $12: 51-13: 03$ & SOHO disabled \\
\hline 2 & 30 Aug. 1998 & $18: 00-18: 12$ & 8319 & M1.3 & 18:00-18:09 & SOHO disabled \\
\hline 3 & 23 Nov. 1998 & 06:10-07:29 & 8384 & $\mathrm{X} 2.2$ & $06: 28-06: 58$ & $\mathrm{SOHO} / \mathrm{LASCO}$ data gap \\
\hline 4 & 4 Jul. 1999 & 08:00-09:00 & 8611 & C6.4 & 08:20-08:44 & 12:06, not halo CME, $335 \mathrm{~km} \mathrm{~s}^{-1}$ \\
\hline 5 & 25 Oct. 1999 & $06: 27-06: 46$ & 8741 & M1.7 & $06: 26-06: 34$ & 14:26, partial halo CME, $511 \mathrm{~km} \mathrm{~s}^{-1}$ \\
\hline 6 & 10 Feb. 2000 & 01:01-02:06 & 8858 & C7.3 & 01:40-02:39 & 02:30, halo CME, $944 \mathrm{~km} \mathrm{~s}^{-1}$ \\
\hline 7 & 23 Mar. 2000 & $11: 30-12: 00$ & 8910 & M2.0 & $11: 32-12: 30$ & $\mathrm{SOHO} / \mathrm{LASCO}$ data gap \\
\hline 8 & 12 Apr. 2000 & 03:29-03:53 & 8948 & M1.3 & $03: 27-03: 50$ & 04:54, not halo CME, $215 \mathrm{~km} \mathrm{~s}^{-1}$ \\
\hline 9 & 25 Aug. 2000 & $14: 22-16: 17$ & 9143 & M1.4 & $14: 21-14: 46$ & 14:54, partial halo CME, $518 \mathrm{~km} \mathrm{~s}^{-1}$ \\
\hline 10 & 21 Mar. 2001 & $00: 31-03: 37$ & 9373 & M1.8 & $02: 28-02: 42$ & 03:06, not halo CME, $331 \mathrm{~km} \mathrm{~s}^{-1}$ \\
\hline 11 & 22 Mar. 2001 & 03:48-05:41 & 9373 & M1.0 & $05: 08-05: 26$ & 05:26, partial halo CME, $674 \mathrm{~km} \mathrm{~s}^{-1}$ \\
\hline 12 & 12 Apr. 2001 & $10: 16-10: 34$ & 9415 & $\mathrm{X} 2.0$ & 09:39-10:49 & 10:31, halo CME, $1184 \mathrm{~km} \mathrm{~s}^{-1}$ \\
\hline 13 & 15 Apr. 2001 & $21: 58-22: 27$ & 9415 & C5.1 & $21: 59-22: 22$ & 24:57, not halo CME, $218 \mathrm{~km} \mathrm{~s}^{-1}$ \\
\hline 14 & 15 Apr. 2001 & $22: 46-23: 14$ & 9415 & - & - & 24:57, not halo CME, $218 \mathrm{~km} \mathrm{~s}^{-1}$ \\
\hline 15 & 13 May 2001 & 02:52-04:30 & 9455 & M3.6 & 02:58-03:08 & 03:50, not halo CME, $434 \mathrm{~km} \mathrm{~s}^{-1}$ \\
\hline 16 & 15 May 2001 & $01: 43-03: 32$ & 9455 & M1.0 & 02:53-03:08 & 02:26, not halo CME, $350 \mathrm{~km} \mathrm{~s}^{-1}$ \\
\hline 17 & 15 Jun. 2001 & 06:20-07:36 & 9502 & C 3.8 & $06: 34-06: 50$ & 06:30, not halo CME, $562 \mathrm{~km} \mathrm{~s}^{-1}$ \\
\hline
\end{tabular}

first review of TRACE observations (Schrijver et al. 1999) it was noted that loop oscillations commonly occur after flare-like processes. In a more detailed study of 17 oscillation events (see Table 1 in Schrijver et al. 2002) a GOES C-, M-, or X-class flare was identified in 16 out of the 17 cases, and a filament destabilisation in at least 3 out of the 17 cases, but no oscillation event occurred without either one. Since flares and destabilised filaments are often seen to be associated with coronal mass ejections (CMEs), we would expect that there can be an intimate connection between CMEs and loop oscillations. Wills-Davey (2007) studied the association of the 23 loop oscillation events of Aschwanden et al. (2002) with CMEs and erupting filaments detected by EIT and identified 12 CME/EIT events and 11 destabilising filaments to be associated with the 23 oscillation events. Moreover, Wills-Davey (2007) finds that destabilising filaments tend to be associated with oscillating loops that have a stable centroid location, while CME events tend to be associated with loop oscillations that have a strong superimposed proper motion. These cases seem to indicate that the CMEs also move the coronal plasma that surrounds the oscillating loops, and this way drag the oscillating loops along, like butterflies that are carried by the wind.

In Table 1 we compile the same 17 oscillation events analysed in Schrijver et al. (2002) and Aschwanden et al. (2002) and list the associated GOES flares and CME events identified from the SOHO/LASCO CME catalog. In 16 out of the 17 cases there is a simultaneous GOES flare identified (see also Table 1 in Schrijver et al. 2002). From the 17 cases we have data gaps from $\mathrm{SOHO} / \mathrm{LASCO}$ in four cases (Gopalswamy, private communication), but in all the 13 other cases we identified a possibly associated CME event within $8 \mathrm{~h}$ of the GOES flare start. The association of CMEs to flares bears some uncertainties, because they are detected earliest in the SOHO/LASCO C2 coronagraph at distances of 1.5-6 solar radii, after a delay in the order of an hour, depending on their speed, acceleration, or deceleration. If we restrict the time delay in our association statistics, we find a CME detection within $3 \mathrm{~h}$ in 11 out of the 13 cases of loop oscillations, or a CME detection within $2 \mathrm{~h}$ in 9 out of 13 cases, respectively. Given the fact that faint or disk-centre CMEs may go undetected in $\mathrm{SOHO} / \mathrm{LASCO}$, we can conjecture that the majority of loop oscillation event is associated or triggered by a flare, CME, or eruptive filament event.

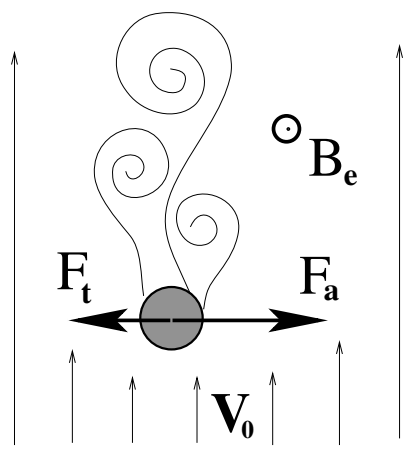

Fig. 1. Excitation of horizontally polarised transverse oscillations of coronal loops by CME upflow. The shaded circle represents the apex segment of the coronal loop, situated perpendicularly to the plane of the sketch. Vertical thin lines show the quasi-steady upflow with the speed $\boldsymbol{V}_{0}$. The external magnetic field $\boldsymbol{B}_{\mathrm{e}}$ is directed parallel to the magnetic field in the loop segment. Interacting with the loop, the flow experiences formation of vortices (curly thin curves), which shed periodically from opposite sides of the loop. In response, the loop is periodically pushed in the horizontal direction by the force $\boldsymbol{F}_{a}$. The loop displacement causes appearance of the restoring MHD force $\boldsymbol{F}_{\mathrm{t}}$. In the resonant case, transverse oscillations of the loop are excited.

\section{Phenomenon of vortex shedding}

Consider the upper, horizontal cylindrical segment of a coronal loop in the presence of an upflow $V_{0}(t)$ representing the eruption (see Fig. 1). The upflow speed is sub-Alfvénic. Initially, the cylinder is in equilibrium. From the point of view of the upflow, the cylinder is an obstacle, or a bluff body, which modifies the flow pattern. It is well-known in hydrodynamics that the interaction of a steady flow with such an obstacle is accompanied by the generation of a sequence of vortices, the Kármán vortex street (see, e.g. Williamson 1996). The vortices are generated periodically downstream alternately from either side of the cylinder. This is known as a hydrodynamic phenomenon of vortex shedding. This phenomenon is more pronounced for high Reynolds number flows, typical e.g. for coronal plasmas. Hence, the cylinder experiences a periodic force which is perpendicular to both the flow velocity and the axis of the cylinder. A common illustration of this effect is the waving of a flag in the wind. Clearly, 
the resonance of the flow-induced oscillations with a natural frequency of the coronal loop would lead to the excitation of the oscillations.

The frequency $f_{a}$ of vortex shedding is connected with the diameter of the cylinder $d$, which is the minor radius of the loop in our context, and the flow speed $V_{0}$ as

$\mathrm{St}=f_{a} d / V_{0}$,

where St is the Strouhal number, which is about $0.15-0.4$ for a very broad range of parameters (Williamson 1996). Thus, for a coronal loop of $1 \mathrm{Mm}$ in diameter and the flow speed $0.1 \mathrm{Mm} / \mathrm{s}$, the period of the vortex shedding oscillations is about one minute, which is comparable to the natural periods of standing kink fast magnetoacoustic modes of coronal loops. As the period of the global kink mode is $2 L / C_{\mathrm{k}}$, where $L$ is the loop length and $C_{\mathrm{k}}$ is the kink speed (see Edwin \& Roberts 1983), the condition for the exact resonance is

$\frac{V_{0}}{C_{\mathrm{k}}}=\frac{1}{2 \mathrm{St}} \frac{d}{L}$.

As the loop diameter is much less than its length, $d \ll L$, the resonant condition can be fulfilled for flow speeds that are much lower than the kink speed.

The force applied to the cylinder from the surrounding flow is proportional to the strength of the vortex and can be expressed as $\boldsymbol{F}_{a}=-\rho_{\mathrm{e}} \boldsymbol{V}_{0} \times \boldsymbol{\Omega}$, where $\rho_{\mathrm{e}}$ is the density of the plasma in the flow, and $\boldsymbol{\Omega}$ the vorticity integrated over the eddy. As in Fig. 1, the generated vorticity is perpendicular to the plane of the sketch and parallel to the axis of the cylinder. The flow is vertical, the force is directed horizontally and perpendicular to the cylinder axis.

An important issue is how the vortex shedding is modified when a magnetic field is present. Full-scale numerical simulations of emerging magnetic flux tubes have demonstrated the importance of this effect and the oscillating ("zigzaging") trajectory of the tube (e.g. Emonet et al. 2001). Dousset \& Pothérat (2008) simulated the interaction of a cylinder with a magnetised flow. It was found that for the field parallel to the axis of the cylinder, the Strouhal number was about 0.2. Thus, it can be taken that, at least in the case when the external magnetic field $\left(\boldsymbol{B}_{0}\right.$ in Fig. 1) is parallel to the axis of the loop segment, the upflow is efficiently accompanied by the vortex shedding phenomenon. On the other hand, if the field in the flow is perpendicular to the axis of the cylinder, magnetic reconnection should be taken into account. The range of parameters in the simulations of Dousset \& Pothérat (2008) was very different from the coronal plasma. However, the phenomenon of vortex shedding has been considered in space plasmas, too. In the ionospheric context the effect of vortex shedding has been intensively studied in connection with $\mathrm{F}$ region bubbles, the field aligned plasma density depletions percolating through the equatorial ionosphere (Ott 1978). The wake of the bubble tends to be a series of vortices alternatively being shed from either side of the bubble, causing a slight rocking of the bubble in the transverse direction. Kereselidze \& Orvelashvili (1987) linked long-period geomagnetic pulsations $P c 5$ with vortex shedding in the solar wind streamlining the Earth's magnetosphere, and estimated the Strouhal number as 0.3. Clear evidence of rolled-up MHD-scale vortices in the magnetosphere has come from multi-spacecraft observations by Cluster (Hasegawa et al. 2004).

The phenomenon of vortex shedding has also been observed in numerical experiments simulating AGN jets (Komissarov 1999). Thus, there are many reasons to expect that this phenomenon can take place in the rarified and magnetised plasmas

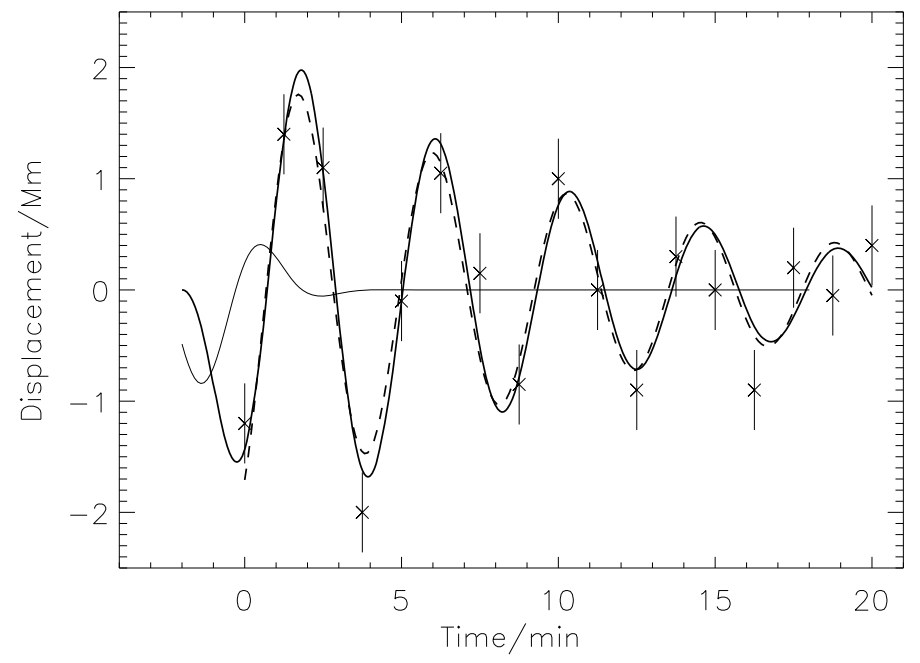

Fig. 2. Comparison of the loop displacement measured in Nakariakov et al. (1999) with the oscillation described by Eq. (3) with the righthand side given by Eq. (4) (solid thick curve). The thin solid curve shows the vortex shedding driving force. The dashed thick curve shows the empirical best fitting exponentially decaying harmonic curve determined in Nakariakov et al. (1999).

of the corona. For example, recently, Chen \& Schuck (2007) has suggested that the decay of vertically polarised kink oscillations of coronal loops could be attributed to aerodynamic drag with zero viscosity and resistivity, which provides the momentum coupling between the oscillating loop and the ambient coronal plasma. It is reasonable to expect both that a coronal loop embedded in the vertical flow should experience a periodic horizontal force due to vortex shedding, at least in the case where the external magnetic field is parallel to the plane of the loop, hence the axis of the generated vorticity, and that this force can be in resonance with natural kink oscillations of the loop.

\section{Excitation of transverse oscillations}

In the presence of the force connected with vortex shedding caused by the CME upflow, the dynamics of the apex of the coronal loop can be modelled by the equation

$\ddot{x}+2 \gamma \dot{x}+\omega_{\mathrm{K}}^{2} x=a(t) \cos \left[2 \pi f_{a}(t) t\right]$,

where $x$ is the transverse displacement of the loop, $\omega_{\mathrm{K}}$ the angular frequency of the fundamental kink mode, and $\gamma$ the dissipation factor. The oscillation is caused by the restoring MHD force $F_{\mathrm{t}}$. The righthand side of the equation represents the vortex shedding force. The frequency $f_{a}$ of the force $F_{a}$ is taken as time dependent, which allows us to account for the change in the CME speed. The time variation of the force amplitude $a(t)$ accounts for the change in the background density, e.g. connected with the dimming, and the conditions for the vortex shedding onset.

The efficiency of the excitation of oscillations by the external force on the righthand side of Eq. (3) depends upon the time dependence of the force. In Fig. 2 we compare the time dependence of the loop oscillation observed by Nakariakov et al. (1999) and the solution of Eq. (3) with $\gamma=0.1 \mathrm{~min}^{-1}$, $\omega_{\mathrm{K}}=1.47 \mathrm{rad} / \mathrm{min}$ (these values correspond to the empirically determined in Nakariakov et al. 1999) and the righthand side

$$
\begin{aligned}
& a(t) \cos \left[2 \pi f_{a}(t) t\right]= \\
& 2.53 \exp \left[-(t+1.5)^{2} / 2.5^{2}\right] \cos [(1.47+t / 300) t+2] .
\end{aligned}
$$


The combination of the parameters of the right hand side is determined by the trial and error method, because of the lack of observational information. We see that the oscillation described by Eq. (3) can agree very well with the experimental data. Moreover, the gradual increase in the oscillation amplitude predicted by our model (see Fig. 2) has been observed in a number of events, e.g. events 1a, 4a, 5c, 7a, 10a, 15a, 16a (and, marginally, 3a and 8a) of Aschwanden et al. (2002).

\section{Discussion}

The discussed mechanism for the excitation of kink oscillations is consistent with their observationally established properties: the link of the oscillations with CMEs and the domination of the horizontal polarisation. The relative rarity of the phenomenon of kink oscillations can be explained by the need for the magnetic field in the CME-dragged plasma to be almost parallel to the loop, otherwise the vortex shedding is suppressed. The selectivity of the phenomenon can be explained by its resonant nature: only the loops with the suitable resonant frequency are efficiently excited by the upflow with a given speed. It is not likely that the observed transverse oscillations are the driven solution with the frequency prescribed by the vortex shedding for the following reasons: the observed periods of oscillations are too close to the natural periods to be completely independent; transverse oscillations remain a rather rare phenomenon, hence the oscillations are not excited every time a loop is embedded in a flow. In addition, the discussed excitation mechanism leads to the predominant excitation of the global kink mode, as the whole loop experiences the perpendicular vortex shedding force during the dimming, which is also consistent with observations. The observational fact that in eight oscillation events the amplitude is initially gradually growing during several periods provides us with additional evidence of resonant excitation. As the average duration of the dimming is a few hours, the time interval of the resonance can be long enough to cause the growth in the amplitude observed during first two or three periods. Also, sufficiently short CME-related pulses can be considered as impulsive drivers that can excite the oscillations non-resonantly.

In our opinion, at least a part of the observed kink oscillation phenomena could be explained in terms of this mechanism. The excitation by a blast wave still remains a realistic alternative.
Observationally, these two mechanisms can be distinguished by the polarisation of the excited oscillations. In the blast wave mechanism, the loops oscillate in the direction parallel to the wave vector, while in the vortex shedding mechanism the oscillations are perpendicular to the flow velocity.

This mechanism can also explain the excitation of the propagating transverse waves in supra-arcades (Verwichte et al. 2005) and of flare-associated winking prominences and filaments.

Acknowledgements. We would like to thank Natchimuthuk Gopalswamy for helpful information on the LASCO CME catalog. We acknowledge data from the Virtual Solar Observatory (VSO) Catalog, provided by the National Solar Observatory (NSO) and Stanford University. We acknowledge the support from ISSI, Bern, Switzerland. TVD acknowledges the EC FP7 grant 220555.

\section{References}

Aschwanden, M. J. 2004, Physics of the Solar Corona (Berlin: Springer Praxis Books)

Aschwanden, M. J., Fletcher, L., Schrijver, C. J., \& Alexander, D. 1999, ApJ, 520,880

Aschwanden, M. J., de Pontieu, B., Schrijver, C. J., \& Title, A. M. 2002, Sol. Phys., 206, 99

Chen, J., \& Schuck, P. W. 2007, Sol. Phys., 246, 145

Dousset, V., \& Pothérat, A. 2008, Phys. Fluids, 20, 017104

Edwin, P. M., \& Roberts, B. 1983, Sol. Phys., 88, 179

Emonet, T., Moreno-Insertis, F., \& Rast, M. P. 2001, ApJ, 549, 1212

Hasegawa, H., Fujimoto, M., Phan, T.-D., et al. 2004, Nature, 430, 755

Hudson, H. S., \& Warmuth, A. 2004, ApJ, 614, L85

Kereselidze, Z. A., \& Orvelashvili, V. S. 1987, Geomagnetism and

Aeronomy/Geomagnetizm i Aeronomiia, 27, 165

Komissarov, S. S. 1999, MNRAS, 308, 1069

Nakariakov, V. M., \& Ofman, L. 2001, A\&A, 372, L53

Nakariakov, V. M., \& Verwichte, E. 2005, Living Rev. Solar Phys., 2, 3, URL(05/07/2005): http : //www . livingreviews . org/lrsp-2005-3

Nakariakov, V. M., Ofman, L., Deluca, E. E., Roberts, B., \& Davila, J. M. 1999, Science, 285, 862

Ott, E. 1978, J. Geophys. Res., 83, 2066

Ofman, L. 2007, ApJ, 655, 1134

Schrijver, C. J., Title, A. M., Berger, M. E., et al. 1999, Sol. Phys., 187, 261

Schrijver, C. J., Aschwanden, M. J., \& Title, A. M. 2002, Sol. Phys., 206, 69

Van Doorsselaere, T., Nakariakov, V. M., \& Verwichte, E. 2008a, ApJ, 676, L73

Van Doorsselaere, T., Nakariakov, V. M., Young, P. R., \& Verwichte, E. 2008b, A\&A, 487, L17

Verwichte, E., Nakariakov, V. M., \& Cooper, F. C. 2005, A\&A, 430, L65

Williamson, C. H. K. 1996, Ann. Rev. Fluid Mech., 28, 477

Wills-Davey, M. 2007, American Astronomical Society Meeting Abstracts, 210, $\# 120.05$ 